0

\title{
Figures of merit for the evaluation of regenerative power converters
}

\author{
Sebastien Maestri, Rogelio Garcia Retegui, Daniel Carrica \\ Universidad Nacional de Mar del Plata, Argentina \\ Stefano Rossini, Gilles Le Godec, Konstantinos Papastergiou \\ CERN, Geneva, Switzerland
}

Keywords: Regenerative power converters, Energy management, Energy recovery, Grid power limitation.

\begin{abstract}
In some applications, like those for particle accelerators and transfer lines, electromagnet loads operate in cycling mode i.e. absorbing or extracting energy from or to a power source. Built-in energy storage such as electrochemical capacitors can be used whose lifetime is heavily depended on the current ripple and depth of discharge. Moreover, the sizing of this storage element is a trade-off between power taken from the grid and energy stored. Additionally, the adopted power conversion structure and control strategy impact on such decision process. In this work, a number of topologies have been evaluated for these applications and the key metrics used to compare them. The proposed figures of merit are used through examples to highlight the trade-offs related with the choice of dc-bus regulation topology and control strategy.
\end{abstract}

Presented at: EPE 2016, 5-9 September 2016, Karlsruhe, Germany

Geneva, Switzerland

November, 2016 


\title{
Figures of merit for the evaluation of regenerative power converters
}

\author{
${ }^{\dagger}$ Sebastian Maestri, ${ }^{\dagger}$ Rogelio Garcia Retegui, ${ }^{\dagger}$ Daniel Carrica, \\ ${ }^{\ddagger}$ Stefano Rossini, ${ }^{\ddagger}$ Gilles LeGodec and ${ }^{\ddagger}$ Konstantinos Papastergiou \\ $\dagger$ UNIVERSIDAD NACIONAL \\ $\ddagger$ EUROPEAN ORGANIZATION FOR \\ DE MAR DEL PLATA \\ L.I.C. \\ Juan B.Justo 4302 \\ Mar del Plata, Argentina \\ Phone: +54 2234816600 \\ Fax: +542234810046 \\ NUCLEAR RESEARCH - C.E.R.N. \\ TE-EPC GROUP \\ 1211 Geneva 23 \\ Geneva, Switzerland \\ Phone: +41 227675549 \\ Fax: +4122766 8301 \\ Email: somaestri@fi.mdp.edu.ar \\ URL: http://www3.fi.mdp.edu.ar \\ Email:k.papastergiou@cern.ch \\ URL: http://www.cern.ch
}

\section{Acknowledgments}

This work has been performed under a research contract between the European Organization for Nuclear Research (CERN) and the Consejo Nacional de Investigaciones Científicas y Técnicas (CONICET), with the support of the European Particle Physics Latin American Network (EPLANET), the Universidad Nacional de Mar del Plata (UNMDP), the Ministerio de Ciencia, Tecnología e Innovación Productiva (MINCYT), and the Agencia Nacional de Promoción Científica y Tecnológica (ANPCYT).

\section{Keywords}

«Regenerative power converters》, «Energy management》, «Energy recovery», «Grid power limitation》.

\begin{abstract}
In some applications, like those for particle accelerators and transfer lines, electromagnet loads operate in cycling mode i.e. absorbing or extracting energy from or to a power source. Built-in energy storage such as electrochemical capacitors can be used whose lifetime is heavily depended on the current ripple and depth of discharge. Moreover, the sizing of this storage element is a trade-off between power taken from the grid and energy stored. Additionally, the adopted power conversion structure and control strategy impact on such decision process. In this work. a number of topologies have been evaluated for these applications and the key metrics used to compare them. The proposed figures of merit are used through examples to highlight the trade-offs related with the choice of dc-bus regulation topology and control strategy.
\end{abstract}

\section{Introduction}

There is a wide range of applications where the load operates in regenerative way, which determines that in a time interval they take power from the input and in another time interval they send power back to the input. Among others, these applications include generation systems [1], traction electrical drives $[2,3]$, electric vehicles $[4,5,6]$ and particle accelerator facilities [7].

A current trend in this area is to supply the energy required by the load from different power inputs: the average power (equal to power losses) is usually supplied by an energy source (external to the system), while the reactive power is supplied by an energy storage element (internal 


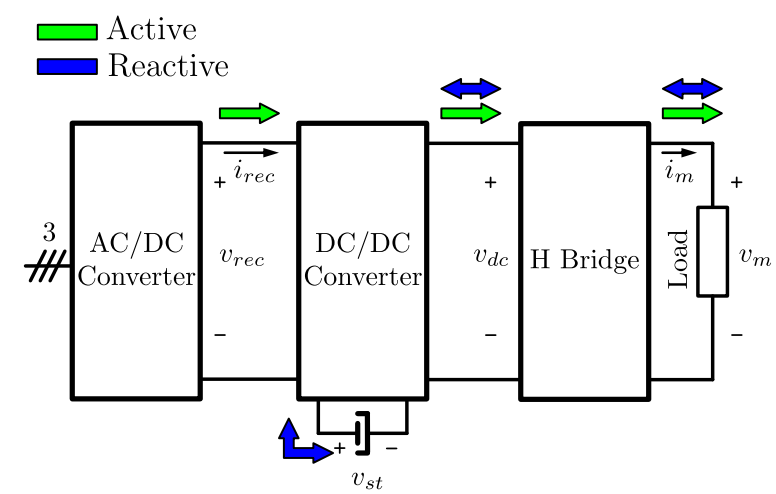

Fig. 1: General structure for regenerative power loads.

to the system) [8]. In general, the former is the electrical network, while the later is commonly implemented with energy storing capacitors for recovering the reactive energy.

Fig. 1 shows a general scheme of a topological structure that allows handling these kind of regenerative loads with input power limitation, where the energy flow between stages is marked with green and blue arrows. The topology includes a grid tied $\mathrm{AC} / \mathrm{DC}$ converter, a $\mathrm{DC} / \mathrm{DC}$ converter, an H-bridge converter and the load. Regarding the AC/DC converter, its output voltage and current are $v_{r e c}(t)$ and $i_{r e c}(t)$, which leads to a power taken from the grid given by $P_{r e c}(t)=v_{r e c}(t) \cdot i_{r e c}(t)$. The DC/DC converter, which includes the energy storage element, is in charge of supplying/receiving the energy between this element and the load. Additionally, it has to vinculate the electrical network and the load in order to supply the active power. The $\mathrm{H}$-bridge is a four-quadrant converter, which allows to supply/receive the energy to the load by generating the corresponding load voltage waveform, $v_{m}(t)$. In this sense, the DC/DC converter output voltage, $v_{d c}(t)$, corresponds to the dc-link voltage of the H-bridge. Notice that the gridtied converter is generally described as an $\mathrm{AC} / \mathrm{DC}$ stage; depending on the implementation of such stage, different power quality issues will arise, like power factor correction or harmonic content. The control of such aspects could be implemented with an additional system, whose analysis is out of the scope of this article.

The division of the instantaneous power to be managed for each structure can come from different motivations, which will be determined by each application. For instance, in [9] is presented a system that uses one energy source (electrical network) and two energy storage elements with different characteristics (a battery having high power with slow dynamics and a capacitor bank with lower power and higher dynamics). In this case, the reactive power is splitted between the energy storage elements, while the energy source could manage the instantaneous active power. However, this cycling operation could produce a remarkable disturbance on the electrical network $[10,11,12]$. In this case, a possibility could be to absorb from the grid a constant power, equal to the average power required by the system; notice that this strategy increases the power supplied/received by the energy storage unit and associated power converter. In any case, there will be a compromise between the energy installed and peak power taken from the electrical network. Consequently, the adopted control strategy for the energy flow will have an impact in the sizing of the elements of the system, especially the energy storage element and the power converters that perform the interface between the energy sources and the load. This is particularly important in the energy storing capacitors stage (denoted as $C_{s t}$ in Fig. 1 ), since its lifetime is tied to, among others, the number of cycles performed or depth of discharge. Additionally, with an approximate electrolytic capacitor cost of $70 € / \mathrm{kJ}$, it becomes interesting to optimise the total energy storage installed.

From the above mentioned, it can be noticed that there will be different structures and control strategies that would allow achieving the objectives required by an application. It is, therefore, interesting to have comparison parameters for helping in both topology and control strategy selection.

This work presents the use of figures of merit for the analysis of power conversion structures that use regenerative loads. A typical application in particle accelerator facilities, where a trapezoidal current profile is applied to an RL load, will be used as study case. Although some of the found expressions might be proper only for applications with this kind of waveforms, it must be noted that the general analysis remains valid for other applications using regenerative loads. 


\section{Application case}

In high-energy physics applications, power converters are used for powering the magnets of the particle accelerators. In particular, the power supplies used in transfer lines, where the particles are delivered from one accelerator to another one, are operated in cycling mode and require the generation of precise high magnetic fields during a given time interval. Fig. 2(a) shows the current profile applied to the magnet load (resistive part $R_{m}$, inductive part $L_{m}$ ), which can be represented by a trapezoidal waveform. The cycle parameters are the flat-top current $\left(I_{R E F}\right)$, the rise time $\left(t_{r}\right)$, the flat-top time $\left(t_{f t}\right)$, the fall time $\left(t_{f}\right)$ and the cycle time $\left(T_{p}\right)$.

The use of power supplies operating at cycling power allows reducing the power consumption from the electrical network. However, this strategy can generate remarkable disturbances on the grid, especially if loads with a high reactive component are used [13, 7]. Fig. 2 (b) illustrates the power profile required by the load, where it can be noticed that it demands energy during rise time and flat-top time, and that sends back energy during the fall time. Then, the system must be able to handle such energy variation. It must be noted that, if the reactive energy of the magnet is recovered, the electrical network should only provide the average power losses, $P_{m \text { avg. }}$.

\section{Figures of merit}

In order to quantify the advantages of a given structure and control strategy, this section presents different figures of merit related to power converter requirements and energy storage sizing.

\section{Energy storage}

The size of the energy storage element $\left(C_{s t}\right)$ is defined by the minimum voltage that the capacitor must withstand to avoid compromising the operation of the system. In case of limiting the input power, in general the minimum voltage occurs at the end of flat-top. Between the beginning of the cycle, $t=0$ and the end of flat-top, $t_{d}=t_{r}+t_{f t}$, the energy provided by the electrical network and by $C_{s t}$ is used for rising the current up to $I_{R E F}$ and to supply the energy related to the active component:

$$
\Delta E_{s t\left[t_{d}\right]}+\Delta E_{g\left[t_{d}\right]}=\Delta E_{m\left[t_{d}\right]}+\Delta E_{a c t\left[t_{d}\right]}
$$

where:

$$
\begin{aligned}
& \Delta E_{s t\left[t_{d}\right]}=\frac{1}{2} C_{s t}\left[V_{s t \max }^{2}-V_{s t \min }^{2}\right] \quad \Delta E_{g\left[t_{d}\right]}=\int_{0}^{t_{d}} i_{r e c}(t) v_{r e c}(t) d t \\
& \Delta E_{m\left[t_{d}\right]}=\frac{1}{2} L_{m} I_{R E F}^{2}=E_{m} \quad \Delta E_{a c t\left[t_{d}\right]}=\int_{0}^{t_{d}} i_{m}^{2}(t) R_{m} d t=I_{R E F}^{2} R_{m}\left(t_{f t}+\frac{t_{r}}{3}\right)
\end{aligned}
$$

Operating with (1), the maximum energy variation in the storage element normalized to the

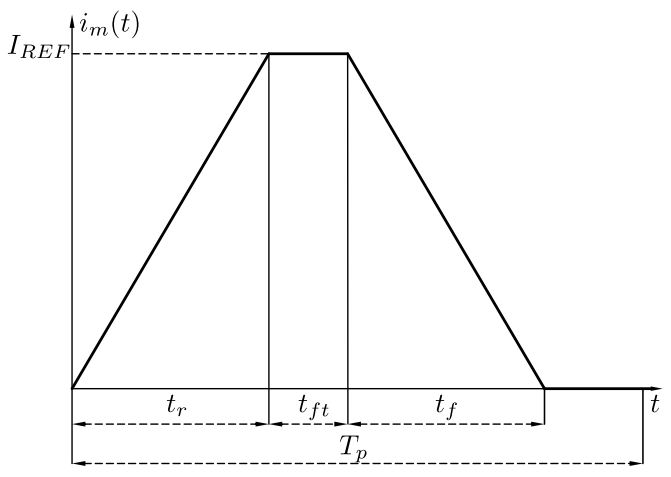

(a)

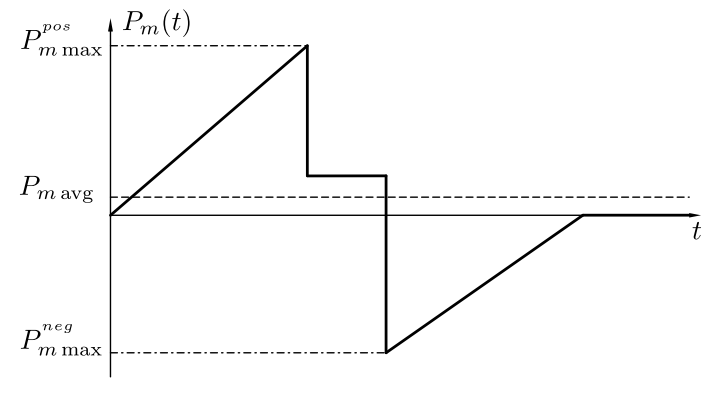

(b)

Fig. 2: (a) Load current waveform. (b) Load power waveform. 
maximum reactive energy can be obtained:

$$
\frac{\Delta E_{s t\left[t_{d}\right]}}{E_{m}}=1+\frac{\Delta E_{a c t\left[t_{d}\right]}-\Delta E_{g_{\left[t_{d}\right]}}}{E_{m}}
$$

Expression (3) represents the energy variation $\frac{\Delta E_{s t}\left[t_{d}\right]}{E_{m}}$ as a function of $\Delta E_{a c t\left[t_{d}\right]}$ and parametric with the amount of energy coming from the electrical grid during $t_{d}, \Delta E_{g}\left[t_{d}\right]$. If it is considered that the main task of the storage element is to provide the reactive energy demanded by the load, this expression indicates how much extra energy must be stored. Notice that this figure of merit depends on the control strategy adopted for the energy flow, i.e. the amount of energy that comes from the electrical network during $t_{d}$ impacts on the energy to be stored. Then, operating with (2) and (3), the minimum value for the capacitor that allows obtaining the required minimum voltage can be obtained:

$$
C_{s t \min }=\frac{2 E_{m}\left(\Delta E_{s t} / E_{m}\right)}{V_{s t \max }^{2}-V_{s t \text { min }}^{2}}
$$

Another important aspect is the efficiency of the installed energy, i.e. how much of the installed energy is really used. Then, the utilisation factor is defined as the energy variation in the energy storage element normalized to its maximum energy:

$$
\begin{aligned}
\frac{\Delta E_{s t}}{E_{s t \max }} & =\frac{(1 / 2) C_{s t}\left(V_{s t \max }^{2}-V_{s t \text { min }}^{2}\right)}{(1 / 2) C_{s t} V_{s t \max }^{2}} \\
\frac{\Delta E_{s t}}{E_{s t \max }} & =1-\left(\frac{V_{s t \min }}{V_{s t \text { max }}}\right)^{2}
\end{aligned}
$$

Notice that this utilisation factor corresponds to the depth of discharge of the energy storage element. In this sense, this figure of merit will impact on the capacitor technology (electrolytic, metalized film, etc) and in the capacitor bank structure to get the required energy (parallel/series combination of capacitor units). Consequently, it will impact both in price and volume.

It can be noticed that this figure improves when the voltage discharge increases and the maximum voltage decreases. Then, this figure of merit is related to the topological structure used, which defines how large can be the voltage discharge. Finally, another important factor is the maximum energy installed normalized to the maximum reactive energy, since it defines the volume of the power supply as a function of the required load. The associated figure of merit can be obtained by operating with (3) and (5):

$$
\begin{aligned}
\frac{E_{s t \text { max }}}{E_{m}} & =\frac{(1 / 2) C_{s t} V_{s t \text { max }}^{2}}{E_{m}}=\frac{\Delta E_{s t}}{E_{m}} \frac{E_{s t \text { max }}}{\Delta E_{s t}} \\
\frac{E_{s t \text { max }}}{E_{m}} & =\frac{\Delta E_{s t} / E_{m}}{\Delta E_{s t} / E_{s t \text { max }}}
\end{aligned}
$$

It could be noticed from (6) that the maximum installed energy decreases when the energy provided by the grid during $t_{d}$ increases and when the efficiency increases.

Regarding the peak power capability of the DC/DC converter, the use of a figure of merit associated to its maximum power related to the maximum power in the load is proposed:

$$
\frac{P_{s t \max }}{P_{m \max }}=\frac{\max \left[v_{s t}(t) i_{s t}(t)\right]}{\max \left[\left\|v_{m}(t) i_{m}(t)\right\|\right]}
$$

It is worth noticing that both $P_{m \max }$ and $P_{s t \max }$ are instantaneous maximum values, then their values could include components of active and reactive power. Consequently, this figure of merit will depend on both the control strategy for the energy flow (i.e., when the grid delivers power and how much is delivered) and on the topology (i.e., how is the distribution of the power inside the $\mathrm{DC} / \mathrm{DC}$ converter). Then, the expressions must be evaluated for each topology taking into account the control strategy selected. 


\section{$\mathrm{AC} / \mathrm{DC}$ power converter}

\section{Power of AC/DC converter}

Considering unitary efficiency in the power converters and that the power losses are mainly given by losses in the load, the energy coming from the grid during the cycle period, $\Delta E_{\left.g_{\left[T_{p}\right]}\right]}$, must be equal to the active energy taken by the load, $\Delta E_{a c t\left[T_{p}\right]}$. Then:

$$
\begin{aligned}
\Delta E_{g\left[T_{p}\right]} & =\Delta E_{a c t\left[T_{p}\right]} \\
\int_{0}^{T_{p}} i_{r e c}(t) v_{r e c}(t) d t & =\Delta E_{a c t\left[T_{p}\right]} \\
\overline{P_{r e c}} T_{p} & =\Delta E_{a c t\left[T_{p}\right]}
\end{aligned}
$$

Finally, the average power in the $\mathrm{AC} / \mathrm{DC}$ converter results:

$$
\overline{P_{r e c}}=\frac{\Delta E_{a c t\left[T_{p}\right]}}{T_{p}}
$$

It must be noticed that $\overline{P_{\text {rec }}}$ represents the minimum power that the $\mathrm{AC} / \mathrm{DC}$ converter must supply, considering that the maximum time $\left(T_{p}\right)$ is used for recovering the power losses $\left(\Delta E_{a c t}\left[T_{p}\right]\right)$. Then, considering that the input power is regulated to keep it lower than certain limit $\left(\hat{P}_{\text {rec }}\right)$, it is assumed that such power will be approximately constant during the time the grid delivers energy, $T_{g}$, where $T_{g} \leq T_{p}$. As a consequence, the amount of energy taken from the electrical network during $T_{g}$ is given by $\Delta E_{g\left[T_{g}\right]}=\Delta E_{a c t\left[T_{p}\right]} \approx \hat{P}_{r e c} T_{g}$, where $\hat{P}_{r e c}$ is peak power in the input. Then, the time $T_{g}$ is modulated as a function of the relationship between the limit input power and the power given by $((8))$. In this sense, the relationship between $\hat{P}_{r e c}$ and $\overline{P_{\text {rec }}}$ is given by:

$$
\frac{\hat{P}_{r e c}}{\bar{P}_{r e c}} \approx \frac{T_{p}}{T_{g}}
$$

Notice that allowing a higher energy from the grid (increasing $\Delta E_{g_{\left[T_{g}\right]}}$ ) will decrease the energy stored, as it is shown in (3) and (6), at the expenses of increasing the peak power taken from the electrical network. Considering that $\overline{P_{r e c}}$ is the minimum power that the AC/DC converter must withstand, the figure of merit represented in (9) express how the AC/DC converter must be oversized. Notice that this figure of merit depends on the control strategy used for regulating the input power.

\section{AC/DC converter output current}

The output current of the AC/DC converter, $i_{r e c}(t)$, will be function of its output voltage and power, $v_{r e c}$ and $P_{r e c}$, respectively:

$$
i_{r e c}(t) v_{r e c}(t)=P_{r e c}(t) \Rightarrow i_{r e c}(t)=\frac{P_{r e c}(t)}{v_{r e c}(t)}
$$

In this sense, the control of the input power could be performed from the control of voltage $v_{r e c}(t)$ or current $i_{\text {rec }}(t)$. Then, the value of the maximum output current of AC/DC converter will depend on the control strategy. In order to obtain the corresponding figure of merit, the maximum output current in the AC/DC converter is normalized to the current obtaind when $P_{r e c}(t)=\overline{P_{r e c}}$ and the voltage $v_{r e c}$ is constant during the cycle period:

$$
\frac{i_{r e c}}{i_{r e c N}}=\frac{\max \left[i_{r e c}(t)\right]}{i_{r e c N}}
$$




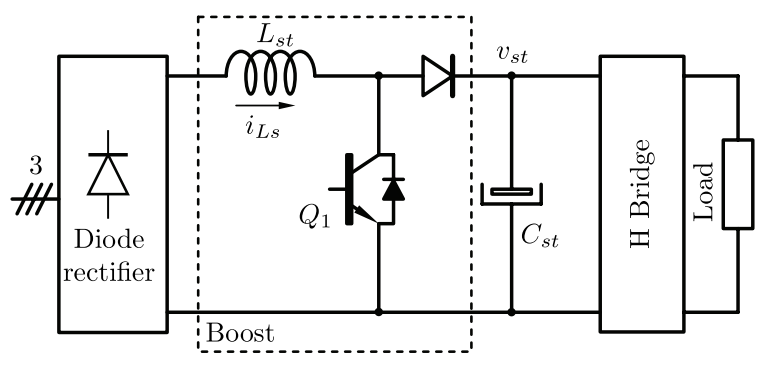

Fig. 3: Topology based on boost front-end converter.

where $i_{r e c N}=\overline{P_{r e c}} / \overline{v_{r e c}}$.

\section{Topology with energy storage}

In this section, a brief description of three topologies with energy storage suitable for their use in the application presented will be performed. These topologies will be used to evaluate the figures of merit.

\section{Boost front-end converter}

A topology based on boost converter is shown in Fig. 3, where it can be noted the different stages of the circuit: diode rectifier, boost converter, H-bridge, and load. The stage given by the $\mathrm{H}$-bridge is used to generate the output current cycle with the required specifications. The stage given by the boost converter has to control the energy flow between the electrical network, the magnet and energy storage capacitor, $C_{s t}$.

It must be noticed that in this topology the storage element voltage corresponds to the dc-link of the H-bridge converter, which must perform the current profile in the magnet. Since the capacitor must deliver/receive energy, its voltage must vary accordingly to the energy flow. One of the effects of this voltage variation in the dc-link of the H-bridge is the change in the open-loop gain of this converter. This effect does not represent a problem, since it can be compensated by a feedforward term proportional to $v_{d c}(t)$ in the regulation loops of the H-bridge control system. Although this effect can be compensated, the minimum voltage in $C_{s t}$ must be bounded by two main factors. The first one is that the boost output voltage should be always greater than the electrical network voltage. The second one is that the minimum boost output voltage must always allow to apply a given voltage in the magnet.

\section{Cascaded structure configuration}

Fig. 4 shows a topology based on a cascaded structure configuration. The input stage is composed by an $\mathrm{AC} / \mathrm{DC}$ converter that is described generally as a capacitor charger, whose main function is to supply a controlled current to a capacitive load (given by $C_{s t}$ ) that varies its voltage between a minimum voltage and a given maximum voltage. This stage should supply a controlled current until the storage voltage reaches the value defined from a reference. In this regard, a linecommutated converter could be a simple and valid alternative for controlling such voltage.

It has to be taken into account that the input current of the capacitor charger will depend on the output current of the charger, input voltage and output voltage (input power equal to output

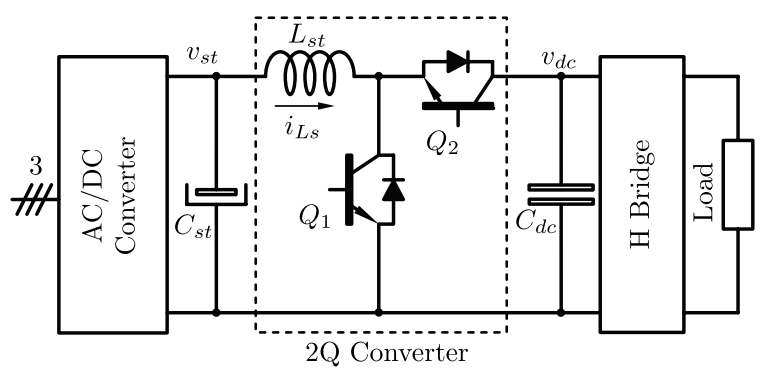

Fig. 4: Topology with cascaded structure configuration. 


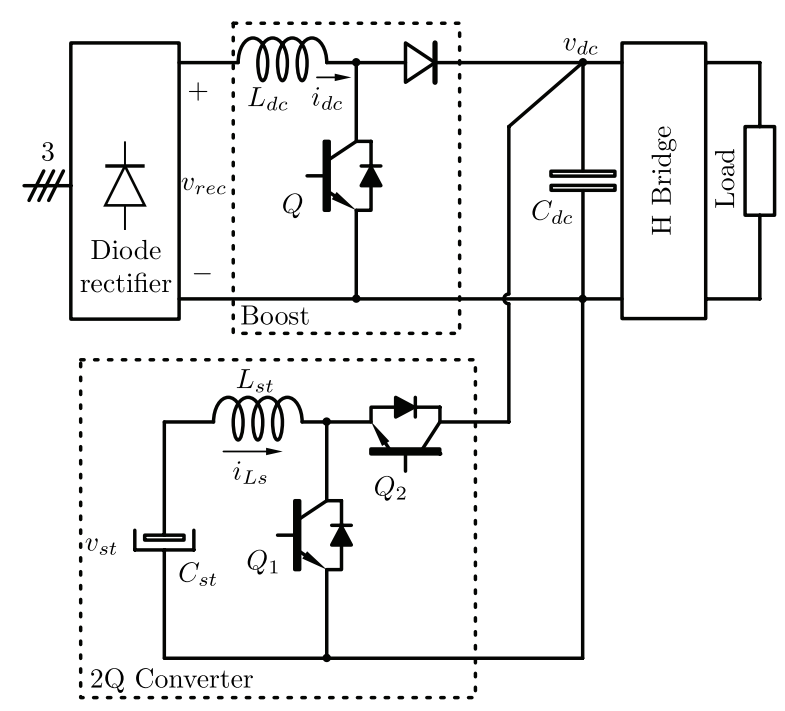

Fig. 5: Topology with parallel structure configuration.

power). The voltages associated to the capacitor charger will be imposed by the system, (the input voltage is the grid voltage and the output voltage is the energy storage element voltage); hence, bounding of the input current must be obtained from the control of such current or the output current of the capacitor charger. Then, considering the variable to be controlled (input current or output current), systems with particular characteristics will be obtained.

The capacitor charger is connected to the energy storage element, which is in turn connected to the dc-link voltage through a bidirectional structure, which is a $2 \mathrm{Q}$ converter. This structure is in charge of managing the energy flow between the energy storage element and the H-bridge. The separation between $C_{s t}$ and $C_{d c}$ allows a wider voltage variation in the storage element and a constant voltage in the dc-link. To allow a higher discharge and to have a higher degree of freedom for defining the maximum storage voltage implies an improvement in the maximum installed energy and utilisation factor. Moreover, a constant voltage in the H-bridge dc-link allows to avoid the problem of the change in the H-bridge gain due to voltage variation and the problem associated to the minimum dc-link voltage required to apply the necessary voltage on the load, which is given by the useful range of the H-bridge duty cycle.

\section{Parallel structure configuration}

Fig. 5 shows a topology based on the parallel connection of two controlled structures. In this topology the grid connected stage is used for supplying the average active power and the storage converter is used for supplying the difference between the electromagnet power and the average active power.

The input stage is formed by a boost converter, whose main task is to supply a controlled current (function of the active power) to the dc-link formed by $C_{d c}$. The control of this current allows to have a high rejection to disturbances in the electrical network voltage. The $2 \mathrm{Q}$ converter is used as coupling stage between the dc-link and the storage element. This converter is bidirectional in current, in order to supply energy from $C_{s t}$ to the H-bridge (during rise time and flat-top time) and to receive energy during the fall time and the time interval when the magnet current is zero. If $C_{s t}$ is considered as the input of the $2 \mathrm{Q}$ converter, it can be seen that this converter behaves like a boost. Hence, the voltage in $C_{d c}$ must be always higher than the voltage in $C_{s t}$. This feature implies that the voltage can vary from values as low as it is practically possible up to the voltage in the dc-link. As it was previously mentioned, this feature is advantageous since it allows to decrease the installed energy and to improve its utilization. Regarding the voltage in $C_{d c}$, it is controlled to a constant value in order to avoid variations in the gain of the H-bridge and to ensure that the load can receive a voltage waveform according to the electromagnet current cycle requirements.

\section{Calculation of figures of merit for topologies}

Table I shows the main parameters of the system that are used for defining the operational conditions and the converter elements. The figures of merit previously presented are evaluated for each topology considering that the power taken from the electrical network is constant 
during the cycle period, i.e. $T_{g}=T_{p}$. Under this consideration and taking into account the values of Table $\mathrm{I}$, the values of the maximum power in the load are $P_{m \max }^{\text {pos }}=236 \mathrm{~kW}$ and $P_{m \text { max }}^{n e g}=-74 \mathrm{~kW}$, while the average power is $P_{m \text { avg }}=15.98 \mathrm{~kW}$. Then, the AC $/$ DC power is $\hat{P}_{r e c}=\overline{P_{\text {rec }}}=15.98 \mathrm{~kW}$. Additionally, a maximum dc-link voltage $v_{d c \max }=700 \mathrm{~V}$ is adopted.

Table I: System parameters for proposed topologies.

\begin{tabular}{cccc||cc}
\hline \multicolumn{3}{c||}{ Pulse parameters } & \multicolumn{2}{c}{ Load parameters } \\
\hline$I_{R E F}$ & $450 \mathrm{~A}$ & $v_{m}$ & $\pm 450 \mathrm{~V}$ & $L_{m}$ & $260 \mathrm{mH}$ \\
\hline$t_{r}$ & $340 \mathrm{~ms}$ & $T_{p}$ & $1.2 \mathrm{~s}$ & $R_{m}$ & $400 \mathrm{~m} \Omega$ \\
\hline$t_{f t}$ & $50 \mathrm{~ms}$ & $t_{f}$ & $220 \mathrm{~ms}$ & & \\
\hline
\end{tabular}

\section{Boost front-end converter}

The maximum storage voltage with the boost front-end converter will be equal to the maximum dc-link voltage $(700 \mathrm{~V})$, while the minimum voltage will be related to the maximum voltage to be applied in the load $(450 \mathrm{~V})$, which occurs when $v_{s t}(t)$ has its minimum value. Additionally, due to the operation of the boost converter, the rectified voltage must be lower than $V_{s t \text { min }}$. Then, $V_{s t \max }=700 \mathrm{~V}, V_{s t \min }=500 \mathrm{~V}$ and $\overline{v_{r e c}}=400 \mathrm{~V}$. Taking into account these data and the previously obtained expressions, the figures of merit indicated in Table II are obtained. Notice that this structure does not require an intermediary converter to vinculate the energy storage with the load; hence, the $P_{s t \text { max }} / P_{m \text { max }}$ figure of merit is zero.

\section{Cascaded structure configuration}

The maximum storage voltage with this topology should be as high as the dc-link voltage, while the minimum voltage will be defined by aspects related to duty cycle/maximum switch current in the 2Q structure (the lower $v_{s t}(t)$, the higher $i_{L s}(t)$ ). Regarding the grid voltage, it will depend on the kind of structure used as grid tied converter. In this case, a thyristor rectifier with maximum rectified voltage equal to $700 \mathrm{~V}$ is used. Then, the following values are adopted: $V_{\text {st } \max }=\overline{v_{\text {rec } \max }}=650 \mathrm{~V}$ and $V_{\text {st min }}=\overline{v_{\text {rec min }}}=200 \mathrm{~V}$.

\section{Parallel structure configuration}

The maximum storage voltage with this topology could be as high as the dc-link voltage, while the minimum voltage will be defined by aspects related to duty cycle/maximum switch current in the $2 \mathrm{Q}$ structure (the lower $v_{s t}(t)$, the higher $i_{L s}(t)$ ). Regarding the rectified voltage, it should lower than the dc-link voltage. Then, the following values are adopted: $V_{s t \max }=650 \mathrm{~V}$, $V_{\text {st } \min }=200 \mathrm{~V}$ and $\overline{v_{\text {rec }}}=400 \mathrm{~V}$.

Taking into account these data and the previously developed expressions, the figures of merit indicated in Table II are obtained.

Table II: Figures of merit for each topology.

\begin{tabular}{cccccccc}
\hline & $\frac{\Delta E_{s t[t d]}}{E_{m}}$ & $\frac{\Delta E_{s t}}{E_{s t \max }}$ & $\frac{E_{s t \max }}{E_{m}}$ & $\frac{P_{s t \max }}{P_{m \max }}$ & $\frac{\widehat{P}_{r e c}}{\bar{P}_{r e c}}$ & $\frac{\widehat{i}_{r e c}}{i_{r e c_{N}}}$ & $C_{s t}$ \\
\hline Boost & 1.266 & 0.49 & 2.58 & 0 & 1 & 1 & $277.7 \mathrm{mF}$ \\
\hline Cascade & 1.266 & 0.91 & 1.4 & 1 & 1 & 2.78 & $174.2 \mathrm{mF}$ \\
\hline Parallel & 1.266 & 0.91 & 1.4 & 1.08 & 1 & 1 & $174.2 \mathrm{mF}$ \\
\hline
\end{tabular}

Table II allows to infer the advantages of one topology over another one. Notice that, since the strategy for input power is the same in all cases, the parameter $\frac{\Delta E_{s t[t d]}}{E_{m}}$ is also equal. Regarding topology comparison, if the size of the energy storage is a priority issue, it can be seen that the boost-based topology is not the more convenient, since it presents the higher value of installed energy due to a low utilisation factor. However, the improvement in the remaining topologies is obtained by increasing the discharge depth, which impacts in the capacitor technology. Then, film capacitors should be used instead of electrolytic, with the consequence of a drastic increase of price and volume. Moreover, between Cascade and Parallel topologies, it can be seen that the differences are related to the DC/DC converter power and the maximum output current of 
the $\mathrm{AC} / \mathrm{DC}$ converter. If a power increase of $10 \%$ is not as critical as an increase in the rectifier output current close to 3 times, the Parallel topology seems to be the most appropiate option.

\section{Conclusions}

This work has presented the use of figures of merit to evaluate power converters for feeding regenerative loads. The figures of merit shown were focused on evaluating aspects related to utilisation of the energy storage element and power ratings of the converters. The usefulness of these metrics was evaluated by comparing three topologies for power supplies suitable for particle accelerator facilities, using the strategy of constant power drawn from the electrical network. The example presented in this work demonstrated that the boost regulator topology requires greater installed energy storage capacity than the other topologies despite its superiority in topological simplicity and better grid-disturbance rejection. Additionally, the energy storage sizing characteristics of both cascaded and parallel converter are similar. However, there are differences in the current ratings of the input power converter, associated to the control strategy used for limiting the input power (the cascaded converter presents an input peak current almost three-times higher). Moreover, the peak power of parallel structure is higher than the cascade converter, which is limited to the peak magnet power.

\section{References}

[1] H. Tao, A. Kotsopoulos, J. Duarte, and M. Hendrix, "Family of multiport bidirectional dc-dc converters," Electric Power Applications, IEE Proceedings -, vol. 153, no. 3, pp. 451-458, May 2006.

[2] F. Ciccarelli, D. Iannuzzi, and D. Lauria, "Supercapacitors-based energy storage for urban mass transit systems," in Power Electronics and Applications (EPE 2011), Proceedings of the 2011-14th European Conference on, Aug 2011, pp. 1-10.

[3] R. Teymourfar, R. Nejati Fard, B. Asaei, and H. Iman-Eini, "Energy recovery in a metro network using stationary supercapacitors," in Power Electronics, Drive Systems and Technologies Conference (PEDSTC), 2011 2nd, Feb 2011, pp. 324-329.

[4] M. Marchesoni and C. Vacca, "New dc ndash;dc converter for energy storage system interfacing in fuel cell hybrid electric vehicles," Power Electronics, IEEE Transactions on, vol. 22, no. 1, pp. 301-308, Jan 2007.

[5] O. Laldin, M. Moshirvaziri, and O. Trescases, "Predictive algorithm for optimizing power flow in hybrid ultracapacitor/battery storage systems for light electric vehicles," Power Electronics, IEEE Transactions on, vol. 28, no. 8, pp. 3882-3895, Aug 2013.

[6] R. de Castro, R. Araujo, J. Trovao, P. Pereirinha, P. Melo, and D. Freitas, "Robust dc-link control in evs with multiple energy storage systems," Vehicular Technology, IEEE Transactions on, vol. 61, no. 8 , pp. $3553-3565$, Oct 2012.

[7] C. Farhni, a. Rufer, F. Bordry, and J. P. Burnet, "A multilevel power converter with integrated storage for particle accelerators," Fourth Power Conversion Conference-NAGOYA, PCC-NAGOYA 2007 - Conference Proceedings, pp. 1480-1483, 2007.

[8] D. Lopez del Moral, A. Barrado, M. Sanz, P. Zumel, C. Raga, A. Lazaro, and H. Miniguano, "Improvement of the synchronous buck converter dynamic performance applied to hybrid electric vehicle regenerative power systems," in Compatibility and Power Electronics (CPE), 2015 9th International Conference on, June 2015, pp. 437-442.

[9] S. Grillo, V. Musolino, L. Piegari, and E. Tironi, "A control strategy for optimizing the power flows supplied by two different storage units," in PowerTech, 2011 IEEE Trondheim, June 2011, pp. 1-6.

[10] C. Fahrni, A. Rufer, F. Bordry, and J. Burnet, "A novel 60 MW pulsed power system based on capacitive energy storage for particle accelerators," in 2007 European Conference on Power Electronics and Applications, vol. 18, no. 4. IEEE, 2007, pp. 1-10.

[11] D. Jovcic and K. Kahle, "Compensation of Particle Accelerator Load Using Converter-Controlled Pulse Compensator," IEEE Transactions on Power Delivery, vol. 21, no. 2, pp. 801-808, Apr. 2006.

[12] E. Bajon, M. Bannon, I. Marneris, G. Danowski, J. Sandberg, and S. Savatteri, "Booster main magnet power supply, present operation and potential future upgrades," in Particle Accelerator Conference (PAC'11), New York, EEUU, 2011.

[13] H. Sato, T. Shintomi, T. Ise, Y. Miura, S. Nomura, and R. Shimada, "Application of Energy Storage System for Stabilization of Accelerator Magnet Power Supply," IEEE Transactions on Applied Superconductivity, vol. 20, no. 3, pp. 1312-1315, Jun. 2010. 